\title{
Effect of shell structure on the nucleon transfer contribution to the imaginary part of the heavy ion optical potential
}

\author{
Fl. Stancu \\ Institute of Physics, University of Liege, B-4000 Liege 1, Belgium \\ D. M. Brink \\ Theoretical Physics Department, University of Oxford, Oxford OX1 3NP, United Kingdom
}

(Received 5 August 1985)

\begin{abstract}
We calculate the imaginary part of the heavy ion optical potential at the strong absorption radius assuming that the absorption is essentially given by the depopulation of the entrance channel due to nucleon transfer. The transfer probabilities of individual nucleons between specific quantum states are calculated with a simple formula. The method is applied to several pairs of nuclei and the results are compared to the experiment and other previous calculations.
\end{abstract}

\section{INTRODUCTION}

In the present work we calculate the imaginary part of the heavy ion optical potential at the strong absorption radius. As pointed out by Broglia, Pollarolo, and Winther, the nucleon transfer process is the dominant mechanism for describing the tail of the imaginary part.

Using such an argument in a previous paper, ${ }^{2}$ we calculated the imaginary part $W_{\text {trans }}$ of the optical potential between various pairs by using the proximity method and a Fermi gas model for describing the interacting nuclei. In this way we could calculate the flux of nucleons tunneling through the barrier formed between the single particle wells. All Coulomb effects were neglected. More recent$1 y^{3}$ the use of the proximity method was formally justified by deriving the definition of $W_{\text {trans }}$ used in Ref. 2 from the transfer probabilities between specific quantum states. The major approximations in relating $W_{\text {trans }}$ to the sum of the transition probabilities were that the nuclei are leptodermous and that their relative motion can be described in a semiclassical approximation by a straight line trajectory.

In the present work we study explicitly the effect of the nuclear structure on the nucleon transfer contribution to the imaginary part. The study is very similar to that of Ref. 4 for $W_{\text {trans }}$, where the shell structure of the interacting nuclei is taken into account but the method is simpler for numerical calculations. The disadvantage is that it is valid only for large separations between nuclei. On the other hand, it can be easily applied to any pair of nuclei. The simplification is due to two main assumptions: (i) The transfer is a peripheral process (the implications of this assumption are discussed in the next section). (ii) The protons and neutrons give identical contributions, as in the proximity method. ${ }^{2}$ This will allow us to compare the present results with those of Ref. 2, which gave good agreement with data.

In the next section we shall derive the formula for $W_{\text {trans. }}$ In Sec. III we present numerical results for various pairs and make a comparison with experiment and other theoretical estimates. Section IV is devoted to discussion and conclusions.

\section{THE FORMALISM}

We assume that the origin of coordinates is located in the center of the target, called nucleus 2 . The projectile, nucleus 1 , moves on a straight line along the $y$ axis with uniform velocity $v$. The distance of closest approach $z=d$ is reached at $t=0$. The transition amplitude $A(2 f, 1 i)$ of a nucleon from a bound state $\psi_{1 i}$ of the projectile to a bound state $\psi_{2 f}$ of the target is given perturbatively by ${ }^{5}$

$$
A(2 f, 1 i) \simeq \frac{1}{i \hbar} \int_{-\infty}^{\infty}\left\langle\psi_{2 f}, V_{1} \psi_{1 i}\right\rangle d t,
$$

where $V_{1}$ is the single particle potential of nucleus 1 . For a peripheral collision this amplitude can take a simpler form. If the separation distance is large enough one can find a plane surface $\Sigma$ at $z=z_{0}$ between the nuclei where both the single particle potentials $V_{1}$ and $V_{2}$ can be neglected for all points $\left(x, y, z_{0}\right)$ at any time $t$. In such a case $A(2 f, 1 i)$ can be rewritten ${ }^{6}$ in terms of a surface integral over $\Sigma$

$$
A(2 f, 1 i) \approx \frac{\hbar}{2 m i} \int_{-\infty}^{\infty} d t \int_{\Sigma} d \mathbf{S}\left(\psi_{2 f}^{*} \nabla \psi_{1 i}-\psi_{1 i} \nabla \psi_{2 f}^{*}\right),
$$

which does not contain single particle potentials any more. This is the basic formula used to evaluate the imaginary optical potential $W_{\text {trans }}$ which can be related to the total transfer probability

$$
P_{b a}=\sum_{i, f}|A(b f, a i)|^{2} \quad(a, b=1,2)
$$

through the relation ${ }^{1,3}$

$$
\frac{2}{\hbar} \int_{-\infty}^{\infty} W_{\text {trans }}[\mathbf{R}(t)] d t=P_{21}+P_{12}
$$

where $\mathbf{R}$ is a point on the trajectory 


$$
\mathbf{R}=\mathbf{d}+\mathbf{v} t .
$$

In order to obtain an analytic expression for $W_{\text {trans }}$ compatible with Eq. (2.4) we have to introduce explicitly the single particle wave functions $\psi_{1 i}$ and $\psi_{2 f}$ in Eq. (2.2). If $\varphi_{i}$ and $\varphi_{f}$ are the eigenstates of the nucleus 1 and 2 at rest corresponding to eigenvalues $\epsilon_{i}$ and $\epsilon_{f}$, respectively, one has

$$
\begin{aligned}
& \psi_{1 i}=\varphi_{i}[\mathbf{r}-\mathbf{R}(t)] e^{(i / \hbar)\left[m \mathbf{v} \cdot \mathbf{r}-\epsilon_{i} t-(1 / 2) m v^{2} t\right]}, \\
& \psi_{2 f}=\varphi_{f}(\mathbf{r}) e^{-(i / \hbar) \epsilon_{j} t} .
\end{aligned}
$$

At this stage we introduce the double Fourier transform

$$
f_{\alpha}\left(k_{x}, k_{y}, z\right)=\int_{-\infty}^{\infty} e^{-i\left(k_{x} x+k_{y} y\right)} \varphi_{\alpha}(x, y, z) d x d y
$$

for $\alpha=i, f$. It can be shown ${ }^{7}$ that if $z$ is outside the range of the single particle potentials, expression (2.8) becomes

$$
f_{i}(z)=C_{i} e^{-\gamma(d-z)} \frac{2 \pi}{\gamma} Y_{l_{i} m_{i}}\left(\hat{\mathbf{k}}_{1}\right) \text { for } \alpha=i
$$

and

$$
f_{f}(z)=C_{f} e^{-\gamma z} \frac{2 \pi}{\gamma} Y_{l_{f} m_{f}}\left(\hat{\mathbf{k}}_{2}\right) \text { for } \alpha=f,
$$

where $C_{\alpha}$ are normalization constants (see the Appendix) and

$$
\begin{aligned}
& \gamma^{2}=k_{x}^{2}+k_{1}^{2}+\gamma_{0 i}^{2}=k_{x}^{2}+k_{2}^{2}+\gamma_{0 f}^{2}, \\
& \hat{\mathbf{k}}_{1}=\frac{1}{\gamma_{0 i}}\left(-i k_{x},-i k_{1}, \gamma\right) ; \hat{\mathbf{k}}_{2}=\frac{1}{\alpha_{0 f}}\left(-i k_{x},-i k_{2}, \gamma\right), \\
& k_{1}=\frac{1}{\hbar v}\left(\epsilon_{f}-\epsilon_{i}-\frac{1}{2} m v^{2}\right) ; k_{2}=\frac{1}{\hbar v}\left(\epsilon_{f}-\epsilon_{i}+\frac{1}{2} m v^{2}\right), \\
& \gamma_{0 \alpha}^{2}=-\frac{2 m}{\hbar^{2}} \epsilon_{\alpha} .
\end{aligned}
$$

Returning to Eq. (2.2) one can see that only the $z$ components of the gradient evaluated at $z=z_{0}$ contribute to the transition amplitude. By replacing $\varphi_{\alpha}$ with the inverse Fourier transform of (2.8) and making the derivative with respect to $z$ of (2.9) and (2.10) one obtains at $z=z_{0}$

$$
\begin{array}{r}
A(2 f, 1 i)=\frac{i \hbar}{m v} \frac{1}{2 \pi} \int_{-\infty}^{\infty} d k_{x} \gamma f_{f}^{*}\left(k_{x}, k_{2}, z_{0}\right) \\
\times f_{i}\left(k_{x}, k_{1}, z_{0}-d\right),
\end{array}
$$

or using the explicit forms (2.9) and (2.10) the transition amplitude from 1 to 2 becomes

$$
\begin{gathered}
A(2 f, 1 i)=i \frac{2 \pi \hbar}{m v} C_{i} C_{f}^{*} \int_{-\infty}^{\infty} d k_{x} \frac{e^{-\gamma d}}{\gamma} Y_{l_{f} m_{f}}^{*}\left(\hat{\mathbf{k}}_{2}\right) \\
\times Y_{l_{i} m_{i}}\left(\hat{\mathbf{k}}_{1}\right) .
\end{gathered}
$$

A similar expression for the transfer of nucleons from the target to the projectile can be obtained by interchanging $i$ and $f$ in (2.16). We notice that $\gamma$ is a function of $k_{x}$, as defined in Eq. (2.11). We introduce the quantity

$$
\eta=\left(k_{1}^{2}+\gamma_{0 i}^{2}\right)^{1 / 2}=\left(k_{2}^{2}+\gamma_{0 f}^{2}\right)^{1 / 2},
$$

which is independent of $k_{x}$ and which will be used below.

The transition probability from a specific orbit $l_{i}$ in nucleus 1 to a specific orbit $l_{f}$ in nucleus 2 is obtained by summing the square modulus of (2.16) over $m_{i}$ and $m_{f}$. Making use of the addition theorem of spherical harmonics, we get

$$
\begin{aligned}
& P_{1 l_{i}, 2 l_{f}}= {\left[\frac{2 \pi \hbar}{m v}\right]^{2} \frac{2 l_{i}+1}{4 \pi} \frac{2 l_{f}+1}{4 \pi}\left|C_{i}\right|^{2}\left|C_{f}\right|^{2} } \\
& \times \int_{-\infty}^{\infty} d k_{x} \int_{-\infty}^{\infty} d k_{x}^{\prime} \frac{e^{-\left(\gamma+\gamma^{\prime}\right) d}}{\gamma \gamma^{\prime}} \\
& \times P_{l_{i}}\left(\hat{\mathbf{k}}_{1}^{*} \cdot \hat{\mathbf{k}}_{1}^{\prime}\right) P_{l_{f}}\left(\hat{\mathbf{k}}_{2}^{*} \cdot \hat{\mathbf{k}}_{2}^{\prime}\right),
\end{aligned}
$$

where

$$
\begin{aligned}
& \hat{\mathbf{k}}_{1}^{*} \cdot \hat{\mathbf{k}}_{1}^{\prime}=\frac{1}{\gamma_{0 i}^{2}}\left(k_{x} k_{x}^{\prime}+k_{1}^{2}+\gamma \gamma^{\prime}\right), \\
& \hat{\mathbf{k}}_{2}^{*} \cdot \hat{\mathbf{k}}_{2}^{\prime}=\frac{1}{\gamma_{0 f}^{2}}\left(k_{x} k_{x}^{\prime}+k_{2}^{2}+\gamma \gamma^{\prime}\right) .
\end{aligned}
$$

We write the transition probability from nucleus 1 to nucleus 2 as

$$
P_{12}=2 \sum_{i, f} \frac{2 j_{i}+1}{2 l_{i}+1} \frac{2 j_{f}+1}{2 l_{f}+1} P_{1 l_{i}, 2 l_{f}} .
$$

This equation contains a number of approximations: (i) We assume that neutrons and protons contribute equally to $P_{12}$. The sum is taken only over neutron states and the factor 2 accounts for the proton contribution. (ii) Actually the transition takes place from levels $j_{i} l_{i}$ to levels $j_{f} l_{f}$. The factors $\left(2 j_{i}+1\right)$ and $\left(2 j_{f}+1\right)$ are the degeneracies of the $j$ levels and occur because we assume that the initial levels $j_{i}$ are full and the final levels $j_{f}$ are empty. (iii) Expression (2.20) contains an implicit dependence on $j_{i}$ and $j_{f}$ through the binding energies $\epsilon_{i}$ and $\epsilon_{f}$. We include this effect by allowing the normalization coefficients $C_{i}$ and $C_{f}$ and $\gamma_{0 i}$ and $\gamma_{0 f}$ to be $j$ dependent. There are other spin-dependent effects which are neglected.

One can evaluate numerically the double integral in (2.18) but it is interesting to go a step further with the analytical expression. If we use the approximations

$$
\begin{aligned}
& \gamma \simeq \eta+\frac{1}{2 \eta} k_{x}^{2}, \quad \gamma^{\prime} \simeq \eta+\frac{1}{2 \eta} k_{x}^{\prime 2} \\
& \frac{1}{\gamma \gamma^{\prime}} \simeq \frac{1}{\eta^{2}}
\end{aligned}
$$

and make the change of variables

$$
x=k_{x}-k_{x}^{\prime} ; X=\frac{1}{2}\left(k_{x}+k_{x}^{\prime}\right) \sqrt{d / \eta},
$$

expression (2.18) becomes 


$$
\begin{aligned}
P_{1 l_{i}, 2 l_{f}=} & \frac{\pi}{2}\left[\frac{\hbar}{m v}\right]^{2}\left(2 l_{i}+1\right)\left(2 l_{f}+1\right)\left|C_{i} C_{f}\right|^{2} \\
& \times \frac{e^{-2 \eta d}}{\eta d} M_{l_{i} l_{f}}
\end{aligned}
$$

where

$$
\begin{aligned}
M_{l_{i} l_{f}}=\frac{1}{\sqrt{\pi}} \int_{-\infty}^{\infty} & d X e^{-X^{2}} P_{l_{i}}\left(A_{i}+B_{i} X^{2}\right) \\
& \times P_{l_{f}}\left(A_{f}+B_{f} X^{2}\right)
\end{aligned}
$$

with

$$
\begin{aligned}
& A_{i}=1+\frac{2 k_{1}^{2}}{\gamma_{0 i}^{2}} ; \quad B_{i}=\frac{2 \eta}{d \gamma_{0 i}^{2}}, \\
& A_{f}=1+\frac{2 k_{2}^{2}}{\gamma_{0 f}^{2}} ; \quad B_{f}=\frac{2 \eta}{d \gamma_{0 f}^{2}} .
\end{aligned}
$$

Approximation (2.23) has been tested numerically and found to be accurate within $1 \%$.

The interesting point about (2.23) is that it shows an explicit exponential decrease of the transition probability and it hints at a similar behavior for $W_{\text {trans }}$. This helps us to find an expression for $W_{\text {trans }}$ compatible with (2.4). Expression (2.23) suggests that each transition will give rise to an absorptive potential of the form

$$
W_{\text {trans }}(R) \sim W_{0} e^{-2 \eta R} .
$$

Around the point of the closest approach one can take

$$
R \simeq d\left(1+\frac{v^{2} t^{2}}{2 d^{2}}\right),
$$

and using it together with (2.26) one can solve the integral on the left-hand side (lhs) of (2.4). This brings us to the following form for the absorptive potential at the strong absorption radius $d$ :

$$
W_{\text {trans }}^{1 i \rightarrow 2 f}(d)=\frac{\hbar v}{2} \sqrt{\eta / \pi d} P_{1 l_{i}, 2 l_{f}},
$$

where we have assumed

$$
W_{\operatorname{trans}}^{1 i \rightarrow 2 f}(d)=W_{0} e^{-2 \eta d} .
$$

The quantity $\eta$ depends on the specific transition, as we shall see in the next section. The imaginary part of the optical potential at the strong absorption radius $d=D_{1 / 2}$ will be calculated as

$$
W_{\mathrm{trans}}(d)=\sum_{i, f}\left[W_{\mathrm{trans}}^{1 i \rightarrow 2 f}(d)+W_{\mathrm{trans}}^{2 i \rightarrow 1 f}(d)\right]
$$

\section{RESULTS}

The essential ingredients of our calculations are the single particle spectra and the normalization constants $C_{\alpha}$ of the asymptotic wave functions of the associated orbits in the initial $\alpha=i$ and the final $\alpha=f$ nuclei.

Each nucleus is described by a single particle potential of the form

$$
V=V_{0} f(r)+V_{l s} \mathbf{1} \cdot \mathbf{S} r_{0}^{2} \frac{1}{r} \frac{d}{d r} f(r),
$$

where

$$
f(r)=\left(1+\exp \frac{r-R}{a}\right)^{-1} .
$$

In order to be able to make a comparison with the proximity results we choose the parameters in Eqs. (3.1)-(3.2) from Bohr and Mottelson ${ }^{8}$ as in Ref. 2,

$$
\begin{aligned}
& V_{0}=-50 \mathrm{MeV}, \quad V_{l s}=22 \mathrm{MeV} ; \\
& r_{0}=1.25, \quad a=0.65 \mathrm{fm}, \quad R=r_{0} A^{1 / 3} .
\end{aligned}
$$

The single particle energies for ${ }^{32} \mathrm{~S},{ }^{60} \mathrm{Ni}$, and ${ }^{208} \mathrm{~Pb}$ and the corresponding normalization constants $C_{\alpha}$ are given in Table I. The $C_{\alpha}$ can also be calculated in the approximation described in the Appendix.

Our results are summarized in Table II. The pairs of nuclei chosen for this study are common either with Ref. 2 or Ref. 4. As a general trend we notice that the present method gives values at the strong absorption radius for $W_{\text {trans }}$ close to those of Pollarolo et al. ${ }^{4}$ On the other hand, they are usually smaller than those obtained with the proximity method, where nuclei are treated in a Fermi gas model. This indicates that the shell structure plays a very important role. In order to see how this effect appears we give in Table III the detailed contribution of each transfer taking place between the pair ${ }^{16} \mathrm{O}+{ }^{40} \mathrm{Ca}$ at $E_{\text {lab }}=74.4 \mathrm{MeV}\left(D_{1 / 2}=9.3 \mathrm{fm}\right)$ and $E_{\mathrm{lab}}=214.1 \mathrm{MeV}$ $\left(D_{1 / 2}=8.8 \mathrm{fm}\right)$. For each transfer we also indicate the value of $\eta$. Equation (2.23) for the transfer probability contains a factor $\exp (-2 \eta d)$, where $d$ is the separation distance between nuclei and $\eta$ is given in Eq. (2.17). Using (2.13) and (2.14) one can rewrite (2.17) as

$$
\eta^{2}=\frac{1}{\hbar^{2} v^{2}}\left[\left(\epsilon_{f}-\epsilon_{i}\right)^{2}+\left(\frac{1}{2} m v^{2}\right)^{2}\right]-\frac{m}{\hbar^{2}}\left(\epsilon_{f}+\epsilon_{i}\right) .
$$

The transfer probability is very sensitive to the value of $\eta$. One can see that a small $Q$ value $\left(Q=\epsilon_{f}-\epsilon_{i}\right)$ at fixed $v$ favors smaller values of $\eta$ and, accordingly, larger values of the transfer probability. This is why levels closest to the Fermi surface usually contribute most to $W_{\text {trans }}$. The quantity $\eta$ also enters the integral (2.24) through the arguments (2.25) and can give a substantial modification to the exponential term in (2.23). The $C_{i}$ and $C_{f}$ in the same equation are sensitive to the binding energy and angular momentum of the corresponding orbits (Table V) and can amplify or diminish the transition probability.

Table II shows several examples of the energy dependence of $W_{\text {trans }}$. We found that it increases in strength with increasing bombarding energy. The single transition values given in Table III show that more channels contribute at higher energies. The same pattern occurs in the other cases. Our examples are in the range where for each pair of levels $\eta$ decreases with increasing energy. This is not always the case. Equation (3.4) shows that for high energies, $\eta$ increases with $v$.

The dependence on $\eta$ also implies a sensitivity of the nucleon transition probability on the single particle spec- 


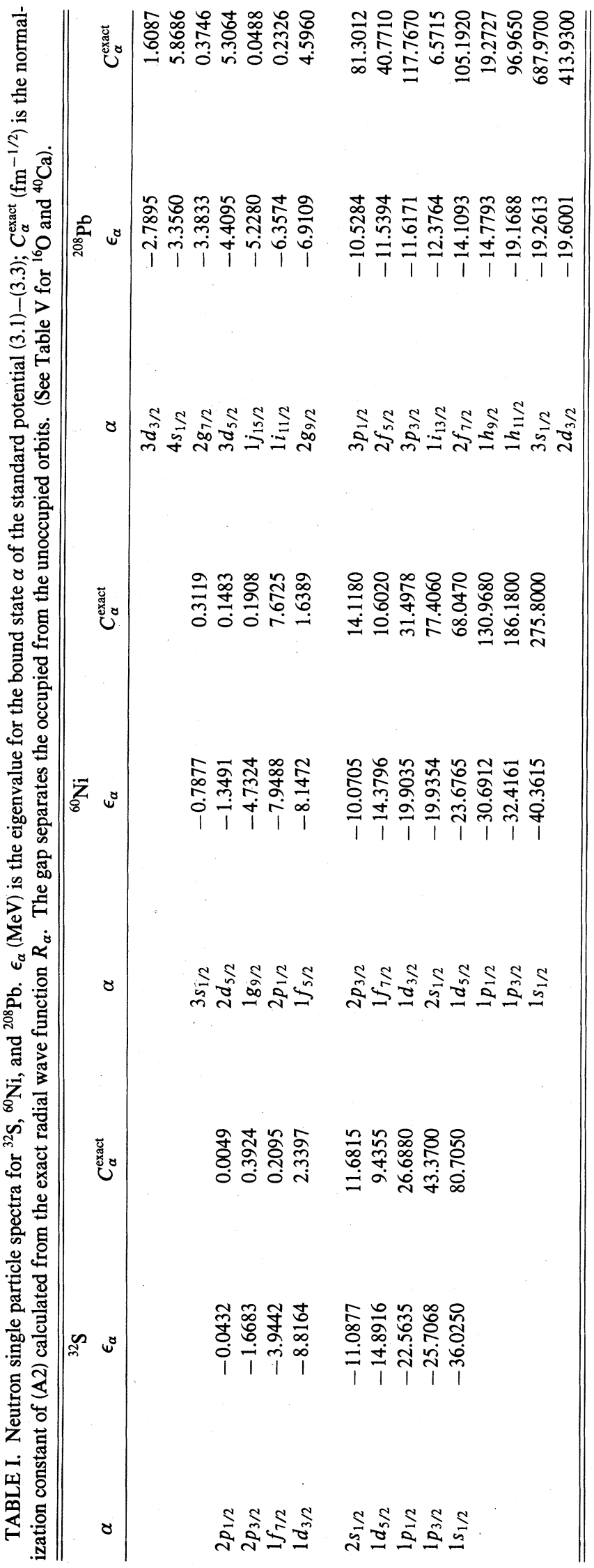

trum associated with each reaction partner. In other words, the results are expected to be sensitive to $\epsilon_{i}$ and $\epsilon_{f}$ of each specific channel through the difference $Q=\epsilon_{i}-\epsilon_{f}$ as well as through the sum $\epsilon_{i}+\epsilon_{f}$, as seen from Eq. (3.4). In order to have an idea of the dependence of the results on the choice made for the single particle spectrum, we give in Table IV the values of the imaginary potential $W_{\text {trans }}^{\text {esps }}$ for ${ }^{16} \mathrm{O}+{ }^{208} \mathrm{~Pb}$ obtained with the experimental single particle spectrum (esps) taken from Ref. 9. The separate contributions from $\mathrm{O} \rightarrow \mathrm{Pb}$ and $\mathrm{Pb} \rightarrow \mathrm{O}$ are also indicated. In these calculations the normalization constants $C_{\alpha}$ have been evaluated according to the approximation described in the Appendix and are based on the standard average potential (3.1)-(3.5) but taking $V_{l s}=0$. The remaining columns in Table IV show the corresponding quantities $W^{\text {app }}$ calculated with theoretical single particle energies. To make a better comparison we have used $C_{\alpha}^{\text {app }}$ obtained by the approximate method. The same number of transitions are included in both cases. In contrast, the values in Table II included contributions from three deeper levels $1 h_{11 / 2}, 3 s_{1 / 2}$, and $2 d_{3 / 2}$, which are not known experimentally. One can see that for all energies the transfer $\mathrm{Pb} \rightarrow \mathrm{O}$ is by far the dominant contribution to $W_{\text {trans, }}^{\text {esps }}$ while in $W_{\text {trans }}^{\text {app }}$ the $\mathrm{O} \rightarrow \mathrm{Pb}$ and $\mathrm{Pb} \rightarrow \mathrm{O}$ transfers contribute more similarly. This change is due to the fact that the experimental occupied single particle spectrum is higher in energy than the theoretical one in ${ }^{208} \mathrm{~Pb}$ and lower in ${ }^{16} \mathrm{O}$. The effect is especially pronounced at $E_{\text {lab }}=129.5 \mathrm{MeV}$.

Finally the largest value of $W_{\text {trans }}^{\text {th }}$ in Table II is for ${ }^{16} \mathrm{O}+{ }^{60} \mathrm{Ni}$. This is because ${ }^{60} \mathrm{Ni}$ is not a closed shell nucleus. The shell effects are therefore less important and the result is closer to the proximity value than for all the other cases. The same holds for the system ${ }^{32} \mathrm{~S}+{ }^{32} \mathrm{~S}$.

\section{CONCLUSIONS}

We have calculated the nucleon transfer contribution to the imaginary part of the heavy ion optical potential by a method which takes account of shell effects. Our results for the absorptive potential at the strong absorption radius are systematically smaller than those obtained with the proximity method ${ }^{2}$ which uses a Fermi gas model. This comparison shows that shell structure plays an important role in the depopulation of the entrance channel.

Our potentials are also smaller than those fitted to experimental data. This is not surprising, as the contribution of inelastic channels has been neglected. The results of Ref. 4 show that this contribution can be important. For example, the inelastic and transfer channels are both significant for ${ }^{16} \mathrm{O}+{ }^{208} \mathrm{~Pb}$ at $E_{\mathrm{lab}}=192 \mathrm{MeV}$.

Sometimes very few transitions contribute to $W_{\text {trans }}$. In the case of the pair ${ }^{16} \mathrm{O}+{ }^{40} \mathrm{Ca}$ at $E_{\text {lab }}=40 \mathrm{MeV}$ there is only one. At $E_{\text {lab }}=214.1 \mathrm{MeV}$ five transitions make a significant contribution (cf. Table III). This illustrates a general trend. At high energies more transitions are important. The energy dependence is influenced strongly by the factor $\exp (-2 \eta d)$ in Eq. (2.23). This factor is a maximum when $\eta$ is as small as possible. In all the cases we have studied $\eta$ decreases with increasing energy. Typical values for the dominant transitions have $\eta$ values lying in 
TABLE II. The absorptive potential due to transfer channels. $D_{1 / 2}$-the strong absorption radius; $W_{\text {trans }}^{\text {exact }}$-the present result; $W_{\text {trans }}^{\mathrm{PBW}}$-from Ref. $4 ; W_{\text {trans }}^{\text {prox }}$-from Ref. $2 ; W^{\exp }$ - the value of the phenomenological potential at the strong absorption radius taken from the references indicated in the last column.

\begin{tabular}{|c|c|c|c|c|c|c|c|}
\hline System & $\begin{array}{c}E_{\text {lab }} \\
(\mathrm{MeV})\end{array}$ & $\begin{array}{l}D_{1 / 2} \\
(\mathrm{fm})\end{array}$ & $\begin{array}{l}W_{\text {trans }}^{\text {exact }} \\
(\mathrm{MeV})\end{array}$ & $\begin{array}{l}W_{\text {trans }}^{\text {PBW }} \\
(\mathrm{MeV})\end{array}$ & $\begin{array}{c}W_{\text {trans }}^{\text {prox }} \\
(\mathrm{MeV})\end{array}$ & $\begin{array}{c}W^{\exp }\left(D_{1 / 2}\right) \\
(\mathrm{MeV})\end{array}$ & Ref. \\
\hline \multirow[t]{5}{*}{${ }^{16} \mathrm{O}+{ }^{40} \mathrm{Ca}$} & 40.0 & 9.7 & 0.0081 & 0.06 & 0.07 & 0.32 & 12 \\
\hline & 55.6 & 9.5 & 0.0648 & & 0.17 & 0.46 & \\
\hline & 74.4 & 9.3 & 0.1605 & 0.18 & 0.31 & 0.66 & \\
\hline & 103.6 & 9.1 & 0.3169 & 0.27 & 0.55 & 0.95 & \\
\hline & 214.1 & 8.8 & 0.4993 & & 1.30 & 1.64 & \\
\hline${ }^{16} \mathrm{O}+{ }^{60} \mathrm{Ni}$ & 142.0 & 9.6 & 0.4693 & & 0.86 & $1.13 \pm 0.17$ & 13 \\
\hline \multirow[t]{3}{*}{${ }^{16} \mathrm{O}+{ }^{208} \mathrm{~Pb}$} & 129.5 & 12.8 & 0.1230 & 0.21 & 0.26 & 0.89 & 12 \\
\hline & 192.0 & 12.4 & 0.2732 & 0.18 & 0.69 & 1.34 & \\
\hline & 312.6 & 12.1 & 0.3143 & & 1.23 & 1.53 & \\
\hline${ }^{32} S+{ }^{32} S$ & 90.9 & 10.1 & 0.0989 & & 0.11 & 0.37 & 12 \\
\hline \multirow[t]{3}{*}{${ }^{40} \mathrm{Ca}+{ }^{40} \mathrm{Ca}$} & 143.6 & 10.75 & 0.0483 & & 0.13 & 0.34 & 14 \\
\hline & 186.0 & 10.72 & 0.1169 & & 0.20 & 0.37 & \\
\hline & 240.0 & 10.6 & 0.2071 & & 0.34 & 0.48 & \\
\hline
\end{tabular}

the range $0.7-1 \mathrm{fm}^{-1}$. This corresponds to a surface diffuseness $a=0.5-0.7 \mathrm{fm}$, consistent with the proximity values in Ref. 2.

For a given $\epsilon_{i}$ the maximum value of $\eta$ occurs when

$$
\epsilon_{f}=\epsilon_{i}+\frac{1}{2} m v^{2},
$$

showing that transitions to the continuum can be significant when the incident energy per nucleon at the Coulomb barrier $\left(\frac{1}{2} m v^{2}\right)$ is of the order of or larger than the separation energy $\left|\epsilon_{i}\right|(\approx 10 \mathrm{MeV})$. For such energies it will not be a good approximation to neglect continuum levels. We note that the proximity method of Ref. 2 treats bound and continuum levels on the same footing. The proximity method should continue to give a reasonable approximation to $W_{\text {trans }}$ even at high energies when the continuum is important.

TABLE III. Detailed contribution of the transitions considered in the calculation of the imaginary potential for ${ }^{16} \mathrm{O}+{ }^{40} \mathrm{Ca}$ at $E_{\mathrm{lab}}=74.4$ and $214.1 \mathrm{MeV}$. For each transition we indicate the value of $\eta$-Eq. (3.4), and the contribution to the imaginary potential calculated with $C_{\alpha}^{\text {exact }}$-Eq. (A2).

\begin{tabular}{rcccc}
\hline \hline $\begin{array}{c}\text { Transition } \\
\mathrm{O} \rightarrow \mathrm{Ca}\end{array}$ & $\eta$ & $E_{\text {lab }}=74.4 \mathrm{MeV}$ & \multicolumn{2}{c}{$E_{\text {lab }}=214.1 \mathrm{MeV}$} \\
\hline $1 p_{1 / 2}-1 f_{7 / 2}$ & 0.7786 & 0.059206 & 0.7943 & $W_{\mathrm{O}-\mathrm{Ca}}^{\text {exact }}$ \\
$-2 p_{3 / 2}$ & 0.8544 & 0.003532 & 0.7718 & 0.064865 \\
$-2 p_{1 / 2}$ & 0.9302 & 0.000126 & 0.7656 & 0.011077 \\
$1 p_{3 / 2}-1 f_{7 / 2}$ & 1.5944 & 0.048211 & 0.001203 \\
$-2 p_{3 / 2}$ & 1.1666 & 0.000248 & 0.9105 & 0.181401 \\
$-2 p_{1 / 2}$ & 1.2652 & 0.000005 & 0.9114 & 0.011468 \\
& & & 0.9189 & 0.001035 \\
$\mathrm{Ca} \rightarrow \mathrm{O}$ & $\eta$ & $W_{\mathrm{Ca}-\mathrm{O}}^{\text {exact }}$ & & \\
\hline $1 d_{3 / 2}-1 d_{5 / 2}$ & 0.8710 & 0.024842 & $\eta$ & $W_{\text {Ca-O }}^{\text {exact }}$ \\
$-2 s_{1 / 2}$ & 0.9390 & 0.000226 & 0.7911 & 0.042559 \\
$2 s_{1 / 2}-1 d_{5 / 2}$ & 0.9501 & 0.013273 & 0.7853 & 0.001304 \\
$-2 s_{1 / 2}$ & 1.0254 & 0.000047 & 0.8265 & 0.089152 \\
$1 d_{5 / 2}-1 d_{5 / 2}$ & 1.1913 & 0.006515 & 0.8244 & 0.002561 \\
$-2 s_{1 / 2}$ & 1.2817 & 0.000010 & 0.9334 & 0.076662 \\
$1 p_{1 / 2}-1 d_{5 / 2}$ & 1.6826 & 0.000002 & 0.9405 & 0.001042 \\
$-2 s_{1 / 2}$ & 1.7885 & $4.3 \times 10^{-10}$ & 1.1521 & 0.005635 \\
$1 p_{3 / 2}-1 d_{5 / 2}$ & 1.8547 & $6.4 \times 10^{-7}$ & 1.1716 & 0.000026 \\
$-2 s_{1 / 2}$ & 1.9638 & $8.3 \times 10^{-12}$ & 1.2294 & 0.009164 \\
$1 s_{1 / 2}-1 d_{5 / 2}$ & 2.4955 & $4.2 \times 10^{-12}$ & 1.2521 & 0.000031 \\
$-2 s_{1 / 2}$ & 2.6125 & $1.2 \times 10^{-16}$ & 1.5203 & 0.000127 \\
\hline \hline
\end{tabular}


TABLE IV. Comparison between results for the imaginary potential of ${ }^{16} \mathrm{O}+{ }^{208} \mathrm{~Pb}$ obtained with the experimental single particle spectrum $W^{\text {esps }}$ and with the theoretical spectrum $W^{\text {app }}$. Separate contributions from transitions $\mathrm{O} \rightarrow \mathrm{Pb}$ and $\mathrm{Pb} \rightarrow \mathrm{O}$ are indicated (see the text for details). In all cases the normalization constants $C_{\alpha}^{\text {app }}$-Eq. (A8)-are used.

\begin{tabular}{llllllll}
\hline \hline $\begin{array}{c}E_{\text {lab }} \\
(\mathrm{MeV})\end{array}$ & $\begin{array}{c}D_{1 / 2} \\
(\mathrm{fm})\end{array}$ & $W_{\mathrm{O} \rightarrow \mathrm{Pb}}^{\text {esps }}$ & $W_{\mathrm{Pb} \rightarrow \mathrm{O}}^{\text {esps }}$ & $W_{\text {trans }}^{\text {esps }}$ & $W_{\mathrm{O} \rightarrow \mathrm{Pb}}^{\mathrm{app}}$ & $W_{\mathrm{Pb} \rightarrow \mathrm{O}}^{\mathrm{app}}$ & $W_{\text {trans }}^{\text {aapp }}$ \\
\hline 129.5 & 12.8 & 0.0021 & 0.3248 & 0.3269 & 0.0336 & 0.0718 & 0.1054 \\
192.0 & 12.4 & 0.0180 & 0.3710 & 0.3890 & 0.0778 & 0.1420 & 0.2198 \\
312.6 & 12.1 & 0.0354 & 0.2552 & 0.2906 & 0.0864 & 0.1240 \\
\hline \hline
\end{tabular}

\section{ACKNOWLEDGMENTS}

We would like to thank L. Lo Monaco for discussions, for checking some of our results, and for help in calculating normalization constants. One of us (F.S.) acknowledges financial support from the Science and Engineering Research Council of the United Kingdom and kind hospitality offered by Peter Hodgson at the Nuclear Physics Department of Oxford University.

\section{APPENDIX}

In this appendix we recall the definition ${ }^{7}$ of the asymptotic normalization constants $C_{\alpha}$ and derive an analytic approximation which can be used for estimating them with a pocket calculator. We introduce $R_{\alpha}(r)$ as the radial part of the eigenstate $\varphi_{\alpha}(\mathbf{r})$ normalized such as

$$
\int_{0}^{\infty}\left|R_{\alpha}(r)\right|^{2} d r=1
$$

The quantity $C_{\alpha}$ is defined as the normalization constant of $R_{\alpha}$ outside the range of the single particle potential. In this region $R_{\alpha}$ can be represented by a spherical Bessel function $h_{l}^{(1)}$ of the third kind ${ }^{10}$ and therefore we can write

$$
R_{\alpha} \simeq-i^{l} C_{\alpha} \gamma_{0 \alpha} r h_{l}^{(1)}\left(i \gamma_{0 \alpha} r\right) \text { for } V \simeq 0 .
$$

The asymptotic form of $h_{l}^{(1)}$ at very large $r$ gives

$$
R_{\alpha} \simeq C_{\alpha} e^{-\gamma_{0 \alpha} r} .
$$

The function $-i h_{l}^{(1)}\left(i \gamma_{0 \alpha} r\right)$ is always positive and we choose by convention to have $C_{\alpha}>0$ always. The exact value of the normalization constant $C_{\alpha}^{\text {exact }}$ can be found by solving the Schrödinger equation numerically for $R_{\alpha}$ and then extracting it from (A2) at an appropriate range $r$. In this case the index $\alpha$ stands for the energy $\epsilon_{\alpha}$ or equivalently the wave number $\gamma_{0 \alpha}$, the angular momentum $l_{\alpha}$, and the total angular momentum $j_{\alpha}$ of the orbit under consideration. Our approximate evaluation of the $C_{\alpha}$ is based on the Wentzel-Kramers-Brillouin (WKB) expression $^{11}$ of the wave function $R_{\alpha}$. If $a_{\alpha}$ and $b_{\alpha}$ are the inner and outer turning points, one can write

$$
R_{\alpha}(r) \sim \frac{\bar{C}_{\alpha}}{\sqrt{\left|\gamma_{\alpha}\right|}} \sin \left[\int_{b_{\alpha}}^{r}\left|\gamma_{\alpha}\left(r^{\prime}\right)\right| d r^{\prime}+\frac{\pi}{4}\right], \quad a_{\alpha}<r<b_{\alpha},
$$

TABLE V. Comparison between $C_{\alpha}^{\text {exact }}$-Eq. (A2)-and $C_{\alpha}^{\text {app }}$-Eq. (A8)-for theoretical single particle levels of ${ }^{16} \mathrm{O}$ and ${ }^{40} \mathrm{Ca}$. We indicate also the binding energy $\epsilon_{\alpha}$, the wave number $\gamma_{0 \alpha}-\mathrm{Eq}$. (2.14), the outer turning point $b_{\alpha}$, and the half-period $\tau_{\alpha}$-Eq. (A11).

\begin{tabular}{lcccccr}
\hline \hline Level & $\begin{array}{c}\epsilon_{\alpha} \\
(\mathrm{MeV})\end{array}$ & $\begin{array}{c}\gamma_{\alpha \alpha} \\
\left(\mathrm{fm}^{-1}\right)\end{array}$ & $\begin{array}{c}C_{\alpha}^{\text {exact }} \\
\left(\mathrm{fm}^{-1 / 2}\right)\end{array}$ & $\begin{array}{c}C_{\alpha}^{\text {app }} \\
\left(\mathrm{fm}^{-1 / 2}\right)\end{array}$ & $\begin{array}{c}b_{\alpha} \\
(\mathrm{fm})\end{array}$ & $\left.\begin{array}{c}\tau_{\alpha} \\
\left(10^{-23}\right.\end{array}\right)$ \\
\hline & & & & & & \\
$2 s_{1 / 2}$ & -2.9373 & 0.3749 & 1.0644 & 2.7440 & 4.9529 & 11.4265 \\
$1 d_{5 / 2}$ & -4.9194 & 0.4852 & 0.5802 & 0.6350 & 3.7505 & 7.1141 \\
& & & & & & \\
$1 p_{1 / 2}$ & -12.2286 & 0.7649 & 4.6102 & 4.2778 & 3.6548 & 6.8580 \\
$1 p_{3 / 2}$ & -17.2769 & 0.9092 & 10.0503 & 5.2666 & 3.3324 & 6.4469 \\
$1 s_{1 / 2}$ & -29.9454 & 1.1970 & 26.8500 & 9.9422 & 2.8892 & 6.7085 \\
& & & & & & \\
$2 p_{1 / 2}$ & -2.1203 & 0.3185 & 0.5804 & 1.7171 & 5.9683 & 12.1040 \\
$2 p_{3 / 2}$ & -4.2638 & 0.4517 & 1.9106 & 3.3520 & 5.5996 & 10.2596 \\
$1 f_{7 / 2}$ & -7.6545 & 0.6052 & 1.2285 & 0.8697 & 4.5320 & 7.3001 \\
& & & & & & \\
$1 d_{3 / 2}$ & -12.8574 & 0.7843 & 6.5208 & 5.7509 & 4.5900 & 7.6115 \\
$2 s_{1 / 2}$ & -14.2045 & 0.8244 & 22.9585 & 18.7093 & 4.8757 & 8.4500 \\
$1 d_{5 / 2}$ & -18.1113 & 0.9309 & 19.3034 & 8.3067 & 4.2586 & 7.3629 \\
$1 p_{1 / 2}$ & -25.6467 & 1.1077 & 46.3880 & 21.8710 & 4.0943 & 7.8970 \\
$1 p_{3 / 2}$ & -28.2255 & 1.1621 & 71.2640 & 22.3218 & 3.9429 & 7.9719 \\
$1 s_{1 / 2}$ & -37.7005 & 1.3430 & 121.2380 & 29.0376 & 3.5469 & 9.5376 \\
\hline \hline
\end{tabular}




$$
\sim \frac{1}{2} \frac{\bar{C}_{\alpha}}{\sqrt{\gamma_{\alpha}}} e^{-w_{\alpha}(r)}, r>b_{\alpha},
$$

where

$$
\begin{aligned}
& \gamma_{\alpha}=\left[\gamma_{0 \alpha}^{2}+\frac{2 m}{\hbar^{2}} V(r)+\frac{\left(l+\frac{1}{2}\right)^{2}}{r^{2}}\right]^{1 / 2}, \\
& w_{\alpha}(r)=\int_{b_{\alpha}}^{r} \gamma_{\alpha}\left(r^{\prime}\right) d r^{\prime} .
\end{aligned}
$$

The normalization constants $\bar{C}_{\alpha}$ can be on one hand related to $C_{\alpha}$. From (A3), (A5), and (A7) one obtains for large $r$

$$
C_{\alpha}=\frac{1}{2} \frac{\bar{C}_{\alpha}}{\sqrt{\gamma_{0 \alpha}}} e^{\gamma_{0 \alpha} b_{\alpha}+K_{\alpha}},
$$

where

$$
K_{\alpha}=\int_{b_{\alpha}}^{r}\left[\gamma_{0 \alpha}-\gamma_{\alpha}\left(r^{\prime}\right)\right] d r^{\prime} ; r \rightarrow \infty .
$$

On the other hand, $\bar{C}_{\alpha}$ can be expressed in terms of the period $2 \tau_{\alpha}$ of the orbit $\alpha$. By making the assumption that the main contribution to the lhs of (A1) comes from the interval $\left(a_{\alpha}, b_{\alpha}\right)$, one has

$$
\int_{0}^{\infty} R_{\alpha}^{2} d r \simeq \frac{1}{2} \bar{C}_{\alpha}^{2} \int_{a_{\alpha}}^{b_{\alpha}} \frac{d r}{\left|\gamma_{\alpha}\right|}=1
$$

where we have averaged over the periodical function. By defining $\tau_{\alpha}$ as

$$
\tau_{\alpha}=\frac{m}{\hbar} \int_{a_{\alpha}}^{b_{\alpha}} \frac{d r}{\left|\gamma_{\alpha}\right|},
$$

one obtains

$$
\bar{C}_{\alpha}^{2}=\frac{2 m}{\hbar} \frac{1}{\tau_{\alpha}}
$$

One can see now that in order to calculate $C_{\alpha}$ from the approximation (A8) one must know $\tau_{\alpha}$ and $K_{\alpha}$. Both these quantities depend on the single particle potential $V(r)$ through (A6). At this stage we neglect the spin-orbit part of (3.1). Then the $j$ dependence of $\tau_{\alpha}$ and $K_{\alpha}$ will come only through $\epsilon_{\alpha}$ (or $\gamma_{0 \alpha}$ ). The half-period $\tau_{\alpha}$ is obtained by integrating (A11) numerically. In $K_{\alpha}$ we make a further approximation, by neglecting also the central part of $V(r)$. Extending the upper limit to $\infty$, the integral (A9) can be evaluated with the result
TABLE VI. Comparison between the imaginary potential calculated with $C_{\alpha}^{\text {app }}$ and $C_{\alpha}^{\text {exact }}$ for separate transitions $\mathrm{O} \rightarrow \mathrm{Ca}$ and $\mathrm{Ca} \rightarrow \mathrm{O}$ at $E_{\text {lab }}=103.6 \mathrm{MeV} ; D_{1 / 2}=9.1 \mathrm{fm}$.

\begin{tabular}{lcc}
\hline Transfer & $\begin{array}{c}W_{\text {trans }}^{\text {app }} \\
(\mathrm{MeV})\end{array}$ & $\begin{array}{r}W_{\text {trans }}^{\text {exact }} \\
(\mathrm{MeV})\end{array}$ \\
\hline $\mathrm{O} \rightarrow \mathrm{Ca}$ & 0.0749 & 0.1965 \\
$\mathrm{Ca} \rightarrow \mathrm{O}$ & 0.0877 & 0.1204 \\
\hline \hline
\end{tabular}

$$
\begin{aligned}
K_{\alpha} \simeq & {\left[\gamma_{0 \alpha}^{2} b_{\alpha}^{2}+\left(l+\frac{1}{2}\right)^{2}\right]^{1 / 2} } \\
& -\left(l+\frac{1}{2}\right) \ln \frac{\left[\gamma_{0 \alpha}^{2} b_{\alpha}^{2}+\left(l+\frac{1}{2}\right)^{2}\right]^{1 / 2}+l+\frac{1}{2}}{\gamma_{0 \alpha} b_{\alpha}} \\
& -\gamma_{0 \alpha} b_{\alpha} .
\end{aligned}
$$

In Table $\mathrm{V}$ we show a comparison between $C_{\alpha}^{\text {exact }}$ obtained from solving the Schrödinger equation for the orbit $\alpha$ and $C_{\alpha}^{\text {app }}$ calculated from (A8) and (A11)-(A14) for ${ }^{16} \mathrm{O}$ and ${ }^{40} \mathrm{Ca}$. Together with the results for $C_{\alpha}$ we also reproduce the values of the quantities $\gamma_{0 \alpha}, b_{\alpha}$, and $\tau_{\alpha}$ used in (A8). We notice that the approximation (A8) takes into account quite well the effect of the barrier (i.e., large values of $C_{\alpha}$ for $l=0$ and smaller values with increasing l). The agreement between $C_{\alpha}^{\text {exact }}$ and $C_{\alpha}^{\text {app }}$ for orbits around the Fermi level is satisfactory. For deeper orbits the agreement worsens because the neglect of $V(r)$ is no longer a good approximation, but the transition probability between these levels is insignificant at the bombardment energies considered throughout this study. For nuclei where $C_{\alpha}^{\text {app }}$ is sometimes larger or sometimes smaller than $C_{\alpha}^{\text {exact }}$, a compensation effect can occur in adding up the contributions of various transitions. A typical example is the transfer $\mathrm{Ni} \rightarrow \mathrm{O}$ at $E_{\text {lab }}=142 \mathrm{MeV}$. But this is not always the case. In Table VI we show results for $W_{\text {trans }}$ of ${ }^{16} \mathrm{O}+{ }^{40} \mathrm{Ca}$ at $E_{\text {lab }}=103.6 \mathrm{MeV}$ obtained on one hand with $C_{\alpha}^{\text {exact }}$ and on the other hand with $C_{\alpha}^{\text {app }}$. We notice that $W_{\mathrm{Ca} \rightarrow \mathrm{O}}$ does not change much but $W_{\mathrm{O} \rightarrow \mathrm{Ca}}$ becomes more than two times larger when $C_{\alpha}^{\text {exact }}$ are used. The approximation (A8) is useful when an exact solution of the Schrödinger equation is not known. For example, transitions between experimental single particle levels can be studied by using experimental energies $\epsilon_{\alpha}$ and by estimating $b_{\alpha}$ and $\tau_{\alpha}$ from the standard single particle potential (3.1)-(3.3). Values of the imaginary potential $W^{\text {esps }}$ in Table IV are calculated in this way.
${ }^{1}$ R. A. Broglia, G. Pollarolo, and A. Winther, Nucl. Phys. A361, 307 (1981).

${ }^{2}$ Fl. Stancu and D. M. Brink, Phys. Rev. C 25, 2450 (1982).

${ }^{3}$ D. M. Brink and Fl. Stancu, Phys. Rev. C 30, 1904 (1984).

${ }^{4}$ G. Pollarolo, R. A. Broglia, and A. Winther, Nucl. Phys. A406, 369 (1983).

${ }^{5}$ D. M. Brink, Les Houches Lectures, edited by R. Balian, M. Rho, and G. Ripka (North-Holland, Amsterdam, 1978), p. 4. ${ }^{6}$ L. Lo Monaco and D. M. Brink, J. Phys. G (to be published).

${ }^{7}$ A. Bonaccorso, G. Piccolo, and D. M. Brink, Nucl. Phys. A441, 555 (1985).
${ }^{8}$ A. Bohr and B. R. Mottelson, Nuclear Structure (Benjamin, New York, 1969), Vol. 1, Sec. 2-4c.

${ }^{9}$ Same as Ref. 8, but Sec. 3-2.

${ }^{10}$ M. Abramowitz and I. A. Stegun, Handbook of Mathematical Functions (Dover, New York, 1965), p. 437.

${ }^{11}$ L. D. Landau and E. M. Lifshitz, Quantum Mechanics (Pergamon, New York, 1965), Chap. VII.

${ }^{12}$ G. R. Satchler and W. G. Love, Phys. Rep. 55, 183 (1979).

${ }^{13}$ G. R. Satchler, Phys. Lett. 58B, 409 (1975).

${ }^{14}$ H. Doubre et al., Phys. Rev. C 15, 693 (1977). 